\title{
Factors predicting poor anticoagulant control on warfarin in a Thai population with non-valvular atrial fibrillation (NVAF): the ACAChE score
}

Piyoros Lertsanguansinchai* ${ }^{*}$, Anurut Huntrakul, Voravut Rungpradubvong,

Ronpichai Chokesuwattanaskul and Somchai Prechawat

\begin{abstract}
Background: In many low- to middle-income countries in Asia, patients with NVAF usually received warfarin for thromboembolic prevention due to cost-effectiveness. The SAMe-TT2R2 score has been proposed to predict TTR in NVAF patients. However, the SAMe-TT2R2 score has not been much validated in Asian population. Interestingly, it may overestimate patients who had TTR $<65 \%$ due to regarding being Asians as a risk factor.

Objectives: To determine the factors predicting poor anticoagulant control on warfarin, create new scoring system, and compare with the SAMe-TT2R2 score in a Thai population with NVAF.

Methods: This is a retrospective study in a tertiary care hospital. We enrolled NVAF patients who received warfarin from January 2014 to December 2018. TTR was calculated based on Rosendaal method. Multiple logistic regression and AUC-ROC curve were used for analysis.
\end{abstract}

Results: A total of 864 patients were enrolled with mean age of $73.6 \pm 11.58$ years. The mean TTR was $48.1 \pm 25.2 \%$. Using multivariate regression analysis, the predictive factors for $\mathrm{TTR}<65 \%$ were antiplatelet use $(\mathrm{OR} 4.49, p \leq 0.001)$, LVEF < 40\% (OR 1.92, $p=0.037)$, chronic kidney disease (GFR $\left.<50 \mathrm{ml} / \mathrm{min} / 1.73 \mathrm{~m}^{2}\right)(\mathrm{OR} 1.68, p=0.013)$, history of CHF (OR 1.7, $p=0.047$ ), and age $\geq 75$ years (OR 1.4, $p=0.037$ ). Based on the regression coefficients, we developed the new scoring system called ACAChE score [A, antiplatelet use (4 points); C, chronic kidney disease, GFR $<50 \mathrm{ml} / \mathrm{min} / 1.73 \mathrm{~m}{ }^{2}$ (2 points); A, age $\geq 75$ years ( 1 point); Ch, history of CHF (2 points); E, LVEF <40\% (2 points)]. ROC curve showed discrimination performance of the ACAChE score and SAMe-TT2R2 score for prediction of TTR $<65 \%$ with the C-statistic of 0.62 (95\% Cl 0.57-0.65) and 0.54 (95\%Cl 0.50-0.58), respectively.

Conclusion: In Thai NVAF patients, the ACAChE score (antiplatelet use, chronic kidney disease (GFR $<50 \mathrm{ml}$ / $\mathrm{min} / 1.73 \mathrm{~m}^{2}$ ), age $\geq 75$ years, history of congestive heart failure, and LVEF $<40 \%$ ) has better prediction for TTR $<65 \%$ than SAMe-TT2R2 score. Thus, it expected to guide the selection of oral anticoagulation in Asian patients with NVAF.

Keywords: Atrial fibrillation, Warfarin, Time in therapeutic range, Same-TT2R2, TTR

*Correspondence: taepiyoros@gmail.com

Division of Cardiology, Faculty of Medicine, Chulalongkorn University and King Chulalongkorn Memorial Hospital, Thai Red Cross, 1873 Rama IV Rd, Pathumwan District, Bangkok 10330, Thailand

\section{Introduction}

Oral anticoagulant is effective in preventing the risk of cardioembolic stroke and reducing mortality in non-valvular atrial fibrillation (NVAF) patients [1]. There was a clear evidence that non-vitamin $\mathrm{K}$ antagonists (NOACs)

c) The Author(s) 2021. This article is licensed under a Creative Commons Attribution 4.0 International License, which permits use, sharing, adaptation, distribution and reproduction in any medium or format, as long as you give appropriate credit to the original author(s) and the source, provide a link to the Creative Commons licence, and indicate if changes were made. The images or other third party material in this article are included in the article's Creative Commons licence, unless indicated otherwise in a credit line to the material. If material is not included in the article's Creative Commons licence and your intended use is not permitted by statutory regulation or exceeds the permitted use, you will need to obtain permission directly from the copyright holder. To view a copy of this licence, visit http://creativeco mmons.org/licenses/by/4.0/. 
significantly reduced incidence of cardioembolic stroke, intracranial hemorrhage, and also mortality. NOACs were associated with lower or similar risk of major bleeding in compared with vitamin $\mathrm{K}$ antagonists (warfarin) in NVAF patients [2-4].

Nowadays, non-vitamin $\mathrm{K}$ antagonist (NOACs) is recommended in many guidelines for stroke prevention in NVAF patients [1,5-7], but warfarin remains the most often prescribed oral anticoagulant drug for stroke prevention, especially in many low- to middle-income countries in Asia due to cost-effectiveness [8].

However, the safety and efficacy of warfarin depend on the average percentage of the time in therapeutic range (TTR) of international normalized ratio (INR) 2.0-3.0 $[9,10]$. The National Institute for Health and Care Excellence guideline recommends that the TTR should not be below $65 \%$ and the European guideline for the Management of AF recommends that TTR should not be below $70 \%$ in AF patients treated with warfarin [1].

Global Anticoagulant Registry in the FIELD-Atrial Fibrillation Registry (GARFIELD-AF registry) showed that there was 2.6-fold increase in the risk of stroke in newly diagnosed AF patients with $\mathrm{TTR}<65 \%$, and also 2.4-fold increase in the risk of all-cause mortality compared with $\mathrm{TTR} \geq 65 \%$ [9].

In 2013, Apostolakis et al. [11] proposed the SAMeTT2R2 score (female sex, 1 point; age $<60$ years, 1 point; medical history (at least 2 of the following: hypertension, diabetes, coronary artery disease/myocardial infarction, peripheral arterial disease, congestive heart failure, previous stroke, pulmonary disease, hepatic or renal disease), 1 point; treatment (interacting drugs), 1 point; current tobacco use, 2 points and non-Caucasian race, 2 points) (Additional file 1: Supplementary table 1) to predict the quality of anticoagulant control with warfarin (as reflected by TTR) and is expected to guide the selection of oral anticoagulation. The SAMeTT2R2 score in the range of 0 to 2 predicts the effect of good anticoagulation control (TTR $\geq 65 \%$ ) and the use of warfarin will be acceptable. If SAMe-TT2R2 score is greater than 2 points, the risk of poor anticoagulation control (TTR $<65 \%)$ increases and the patients may be candidate for the use of NOACs. Non-Caucasian race is immediately assigned for 2 points in this score, so SAMeTT2R2 score in Asian population tends to be high and may overestimate the number of NVAF patients who had TTR $<65 \%$. The validation of the SAMe-TT2R2 score was mainly studied in the Caucasian population [12-16].

However, data of the SAMe-TT2R2 score are limited and have not been much validated in Asian population. Interestingly, it may overestimate the number of NVAF patients who had $\mathrm{TTR}<65 \%$ due to regarding being Asians as an important risk factor.
Our primary objective is to determine the factors for predicting poor anticoagulant control on warfarin. Secondary objective is to create new scoring system, validate the SAMe-TT2R2 score, and compare the new scoring system with the SAMe-TT2R2 score for predicting poor anticoagulant control in Thai population with NVAF who received warfarin.

\section{Methods \\ Study design}

This single-center retrospective cohort study was conducted in King Chulalongkorn Memorial Hospital, Bangkok, Thailand. The protocol for this study was approved by the institutional review board of King Chulalongkorn Memorial Hospital (IRB No. 105/62).

\section{Study population and protocol}

We enrolled consecutive patients with non-valvular atrial fibrillation diagnosed by EKG and Holter monitoring who were treated with warfarin and age more than 18 years old in King Chulalongkorn Memorial Hospital from January 1, 2014, to December 31, 2018, via a computerized database. Enrolled patients must had been using at least 6 months of warfarin therapy, with at least 3 INR measurement. Patients were excluded from this study if they had significant valvular heart disease including prosthetic valve, moderate to severe rheumatic mitral stenosis, interrupted taking warfarin continuously for more than 1 week, INR less than 3 measurement or loss to follow-up.

The percentage of time that a patient had an INR range between 2 to 3 was calculated using the TTR by Rosendaal method. In this study, we defined poor anticoagulant control with warfarin as TTR $<65 \%$. The INR measured within first 30 days after initiation of warfarin did not use to calculate TTR because INR was not constant and fluctuated in the initial phase of warfarin adjustment. Clinical follow-up was performed at least 1 year and up to 4 years by visiting to heart clinic, reviewing of hospital electronic medical records system or by telephone interview.

The data from patient's medical records were collected including baseline clinical characteristics such as sex, age, body mass index, occupation, education, medical history, medication, history of alcohol drinking, history of smoking, previous history of bleeding, left ventricular ejection fraction (LVEF) and laboratory tests (kidney function, liver function, and INR) and SAMe-TT2R2 score. Ischemic stroke risk was calculated at baseline using CHA2DS2-VASc score (Additional file 1: Supplementary table 2). Bleeding risk was calculated at baseline using HASBLED score (Additional file 1: Supplementary table 3 ). Major and minor bleeding events were 
categorized, defined by the 2005 International Society on Thrombosis and Hemostasis (ISTH) criteria (Additional file 1: Supplementary table 4).

\section{Statistical analysis}

Categorical data were presented as frequency and percentage. Continuous data were presented as mean \pm standard deviation (SD) for normal distribution, and as median for skewed distribution.

Categorical data were compared using Chi-square test, and continuous unpaired data were compared using Student's t test.

Univariate and multivariate logistic regression analysis was used to identify factors associated with poor anticoagulant control (TTR $<65 \%)$ on warfarin. Factors with a $p$ value $<0.2$ in univariate analysis were included in multivariate analysis and presented with adjusted odds ratio and $95 \%$ CI. A $p$ value less than 0.05 was regarded as being statistically significant.

Statistically significant factors from final multivariate analysis were converted to the new scoring system, and points were assigned to each factor approximately proportional to magnitude of the regression coefficients.

The discrimination performance of the new scoring system in comparison with the SAMe-TT2R2 score for TTR $<65 \%$ was evaluated by area under a receiver-operating characteristic (ROC) curve. The C-statistic was calculated using a receiver-operating characteristic (ROC) curve for comparison of the discrimination performance.

We also reported the sensitivity, specificity, positive predictive value (PPV), negative predictive value (NPV), positive likelihood ratio, negative likelihood ratio, and odd ratio (OR) for each point of new scoring system and SAMe-TT2R2 score. Clinical implication and Youden's index were performed for selecting appropriate cut point of the new scoring system and the SAMe-TT2R2 score in predicting poor anticoagulant control on warfarin in Thai population with NVAF. $p$ Value $<0.05$ was accepted as statistically significant. All statistical analyses were performed using SPSS version 22.0 statistical software.

\section{Results}

\section{Baseline characteristics}

From January 1, 2014, to December 31, 2018, a total of 889 consecutive non-valvular atrial fibrillation patients (NVAF) who were treated with warfarin at King Chulalongkorn Memorial Hospital, Bangkok, Thailand, were enrolled in this study. A total of 25 patients (2.8\%) were excluded from this study, interrupted taking warfarin continuously for more than 1 week or treatment changed in 13 patients (1.5\%), insufficient INR tests (less than 3 measurement) in 7 patients $(0.8 \%)$, and loss to follow-up data in 5 patients $(0.5 \%)$. A total of
864 NVAF patients who were treated with warfarin had complete data for TTR calculation and were included in this study (Fig. 1).

The average age was $73.6 \pm 11.58$ years and $51.7 \%$ were female. $87.5 \%$ of patients in this study were older than 60 years old. $22.1 \%$ of the patients had tertiary education, and $50 \%$ of the patients in this study had primary or no education. Most patients were nonsmoker (68.9\%), and only $5.32 \%$ of the patients were currently smoking. Most comorbidities were hypertension (78.9\%), dyslipidemia (53.6\%), diabetes mellitus type $2(39.4 \%)$, chronic kidney disease (GFR $<60 \mathrm{ml} /$ $\left.\mathrm{min} / 1.73 \mathrm{~m}^{2}\right)(38.8 \%)$, and coronary artery disease (23.6\%). 86.2\% of the patients had more than one comorbidity. $60.8 \%$ of the patients received medications that had drug interaction with warfarin. Most of medications that had drug interaction with warfarin in this study were statin, antiplatelet, antiarrhythmic drug and non-dihydropyridine calcium channel blocker, respectively. The average left ventricular ejection fraction (LVEF) was $59.2 \pm 14.8 \%$, and $11.5 \%$ of the patients had LVEF $<40 \%$. Most patients received beta-blocker (68.3\%) and only $6.3 \%$ of the patients received amiodarone. $14 \%$ of patients received warfarin with antiplatelet. The average CHA2DS2VASc score, HASBLED, and SAMe-TT2R2 score were $3.9 \pm 1.6,2.77 \pm 1.09$ and $3.1 \pm 0.8$, respectively. The average number of INR measurement was $20.38 \pm 14.44$ tests. The mean TTR was $48.11 \pm 25.18$ and $73.7 \%$ had TTR $<65 \% .8 .4 \%$ of the patients had history of bleeding, $2.1 \%$ of the patients had major bleeding and $6.3 \%$ had minor bleeding. Baseline characteristics of the patients with TTR $\geq 65 \%$ and patients with TTR $<65 \%$ are shown in Table 1 .

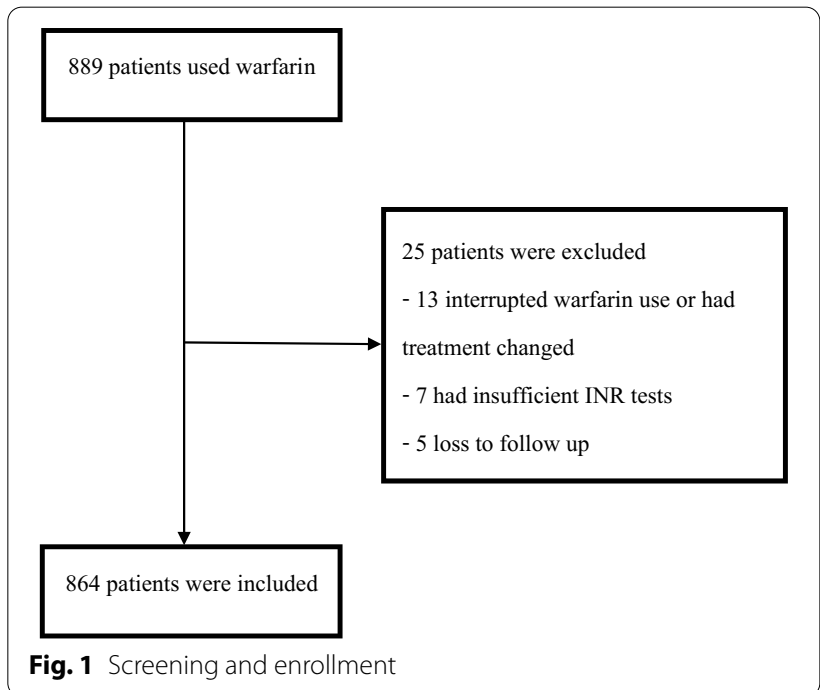


Table 1 Baseline characteristics

\begin{tabular}{|c|c|c|c|c|}
\hline & $\begin{array}{l}\text { All patient }(n=864) \\
n(\%) \mid \text { Mean } \pm \text { SD }\end{array}$ & $\begin{array}{l}\text { TTR } \geq 65 \%(n=227) \\
n(\%) \mid \text { Mean } \pm \text { SD }\end{array}$ & $\begin{array}{l}\text { TTR }<65 \%(n=637) \\
n(\%) \mid \text { Mean } \pm \text { SD }\end{array}$ & $p$ value $^{1}$ \\
\hline Age $<75$ years $\neq$ & $428(49.54)$ & $130(57.27)$ & $298(46.78)$ & $0.007 \neq$ \\
\hline Mean Age (years) & $73.6 \pm 11.58$ & $72.25 \pm 11.96$ & $74.08 \pm 11.41$ & $0.040 \neq$ \\
\hline Female & $447(51.74)$ & $119(52.42)$ & $328(51.49)$ & 0.809 \\
\hline $\mathrm{BMI}\left(\mathrm{kg} / \mathrm{m}^{2}\right)$ & $25.28 \pm 8.54$ & $26.44 \pm 14.4$ & $24.86 \pm 4.96$ & $0.016 \neq$ \\
\hline Education & & & & 0.120 \\
\hline Primary or no & $432(50)$ & $102(44.93)$ & $330(51.81)$ & \\
\hline Secondary & $241(27.89)$ & $65(28.63)$ & $176(27.63)$ & \\
\hline Tertiary or higher & $191(22.11)$ & $60(26.43)$ & $131(20.57)$ & \\
\hline Occupation & & & & 0.219 \\
\hline Government/state enterprises & $145(16.78)$ & $48(21.15)$ & $97(15.23)$ & \\
\hline Employee & $171(19.79)$ & $45(19.82)$ & $126(19.78)$ & \\
\hline Business & $127(14.7)$ & $31(13.66)$ & $96(15.07)$ & \\
\hline Others & $421(48.73)$ & $103(45.37)$ & $318(49.92)$ & \\
\hline Smoking & & & & 0.736 \\
\hline Non-smoker & $595(68.87)$ & $152(66.96)$ & $443(69.54)$ & \\
\hline Current smoker & $46(5.32)$ & $12(5.29)$ & $34(5.34)$ & \\
\hline Ex-smoker & $223(25.81)$ & $63(27.75)$ & $160(25.12)$ & \\
\hline Alcohol & & & & 0.309 \\
\hline Non-drink & $635(73.5)$ & $163(71.81)$ & $472(74.1)$ & \\
\hline Current drink & $20(2.31)$ & $3(1.32)$ & $17(2.67)$ & \\
\hline Ex-drink & 209 (24.19) & $61(26.87)$ & $148(23.23)$ & \\
\hline Herbal use & $36(4.17)$ & $8(3.52)$ & $28(4.4)$ & 0.573 \\
\hline Hypertension & $682(78.94)$ & $181(79.74)$ & $501(78.65)$ & 0.730 \\
\hline Diabetes mellitus & $340(39.44)$ & 75 (33.19) & $265(41.67)$ & $0.025 \neq$ \\
\hline Dyslipidemia & $463(53.59)$ & $112(49.34)$ & $351(55.1)$ & 0.135 \\
\hline Coronary artery disease & $204(23.61)$ & $47(20.7)$ & $157(24.65)$ & 0.230 \\
\hline Peripheral arterial disease & $38(4.4)$ & $12(5.29)$ & $26(4.08)$ & 0.447 \\
\hline Heart failure & $129(14.93)$ & $20(8.81)$ & $109(17.11)$ & $0.003 \neq$ \\
\hline Transient ischemia attack & $78(9.04)$ & $17(7.49)$ & $61(9.59)$ & 0.343 \\
\hline Hepatic impairment & $157(18.17)$ & $30(13.22)$ & $127(19.94)$ & 0.024 \\
\hline CKD (mean GFR ml/min/1.73 m²) & $64.18 \pm 23.44$ & $68.64 \pm 20.3$ & $62.58 \pm 24.28$ & $<0.001 \neq$ \\
\hline CKD (GFR< $\left.60 \mathrm{ml} / \mathrm{min} / 1.73 \mathrm{~m}^{2}\right)$ & $335(38.77)$ & $61(26.87)$ & $274(43.01)$ & $<0.001 \neq$ \\
\hline CKD (GFR $\left.<50 \mathrm{ml} / \mathrm{min} / 1.73 \mathrm{~m}^{2}\right)$ & $222(25.69)$ & $37(16.3)$ & $185(29.04)$ & $<0.001 \neq$ \\
\hline Thrombosis related & & & & 0.053 \\
\hline Systemic emboli & $95(11.01)$ & $22(9.69)$ & $73(11.48)$ & \\
\hline Myocardial infarction & $72(8.34)$ & $11(4.85)$ & $61(9.59)$ & \\
\hline Bleeding & & & & $0.011 \neq$ \\
\hline Major bleeding & $18(2.09)$ & $4(1.76)$ & $14(2.21)$ & \\
\hline Minor bleeding & $54(6.27)$ & $5(2.2)$ & $49(7.73)$ & \\
\hline LVEF (\%) & $59.18 \pm 14.81$ & $61.94 \pm 12.64$ & $58.2 \pm 15.4$ & $<0.001 \neq$ \\
\hline LVEF $<40 \%$ & $99(11.47)$ & $14(6.19)$ & $85(13.34)$ & $0.004 \ddagger$ \\
\hline Cordarone & $55(6.37)$ & $12(5.29)$ & $43(6.76)$ & 0.435 \\
\hline Flecainide & $2(0.23)$ & $0(0)$ & $2(0.31)$ & 1.000 \\
\hline Digoxin & $104(12.04)$ & $26(11.45)$ & $78(12.24)$ & 0.753 \\
\hline Betablocker & $590(68.29)$ & $161(70.93)$ & $429(67.35)$ & 0.320 \\
\hline Non-dihydropyridine CCB & $11(1.27)$ & $0(0)$ & $11(1.73)$ & 0.076 \\
\hline Dihydropyridine CCB & $214(24.8)$ & $52(22.91)$ & $162(25.47)$ & 0.442 \\
\hline Antiplatelet & $121(14)$ & $11(4.85)$ & $110(17.27)$ & $0.001 \neq$ \\
\hline Warfarin (mg/week) & $18.54 \pm 7.74$ & $19.72 \pm 8.31$ & $18.12 \pm 7.49$ & $0.007 \neq$ \\
\hline
\end{tabular}


Table 1 (continued)

\begin{tabular}{|c|c|c|c|c|}
\hline & $\begin{array}{l}\text { All patient }(n=864) \\
n(\%) \mid \text { Mean } \pm \text { SD }\end{array}$ & $\begin{array}{l}\text { TTR } \geq 65 \%(n=227) \\
n(\%) \mid \text { Mean } \pm \text { SD }\end{array}$ & $\begin{array}{l}\text { TTR }<65 \%(n=637) \\
n(\%) \mid \text { Mean } \pm \text { SD }\end{array}$ & $p$ value $^{1}$ \\
\hline Drug interaction & $524(60.86)$ & $142(62.83)$ & $382(60.16)$ & 0.479 \\
\hline INR measurement & $20.38 \pm 14.44$ & $18.73 \pm 11.21$ & $20.96 \pm 15.39$ & $0.021 \neq$ \\
\hline Mean TTR & $48.11 \pm 25.18$ & $78.48 \pm 10.65$ & $36.5 \pm 18.58$ & $<0.001 \neq$ \\
\hline SAME TT2R2 score & & & & 0.135 \\
\hline 2 & $216(25)$ & $65(28.63)$ & $151(23.7)$ & \\
\hline 3 & $365(42.25)$ & $100(44.05)$ & $265(41.6)$ & \\
\hline 4 & $248(28.7)$ & $52(22.91)$ & $196(30.77)$ & \\
\hline$\geq 5$ & $35(4.05)$ & $10(4.41)$ & $25(3.92)$ & \\
\hline Mean CHA2DS2-VASc score & $3.94 \pm 1.62$ & $3.59 \pm 1.63$ & $4.06 \pm 1.60$ & $<0.001 \neq$ \\
\hline Mean HASBLED score & $2.77 \pm 1.09$ & $1.95 \pm 0.97$ & $3.06 \pm 0.97$ & $<0.001 \neq$ \\
\hline HASBLED score $<3$ & $320(37.04)$ & $162(71.37)$ & $158(24.8)$ & $<0.001 \neq$ \\
\hline
\end{tabular}

${ }^{1} p$ value for comparison between patients with TTR $\geq 65 \%$ and TTR $<65 \%$

${ }^{\ddagger} p$ value $<0.05$

Abbreviations: BMI: Body mass index; CKD: Chronic kidney disease; LVEF: Left ventricular ejection fraction; CCB: Calcium channel blocker; INR: International normalized ratio

\section{Factors for prediction of poor anticoagulant control on warfarin (TTR<65\%)}

A total of 637 patients $(73.7 \%)$ in this study had poor anticoagulant control on warfarin (TTR $<65 \%$ ). By using univariate analysis, age $\geq 75$ years $(\mathrm{OR}=1.53$, 95\% CI $=1.12-2.07, p=0.007)$, diabetes mellitus (DM) $(\mathrm{OR}=1.44,95 \% \mathrm{CI}=1.05-1.98, p=0.02)$, congestive heart failure $(\mathrm{OR}=2.14,95 \% \mathrm{CI}=1.29-3.53, p=0.003)$, hepatic impairment $(\mathrm{OR}=1.64,95 \% \mathrm{CI}=1.06-2.52$, $p=0.02)$, chronic kidney disease with GFR $<50 \mathrm{ml} /$ $\mathrm{min} / 1.73 \mathrm{~m}^{2}(\mathrm{OR}=2.10,95 \% \mathrm{CI}=1.42-3.11, p<0.001)$, $\mathrm{LVEF}<40 \%(\mathrm{OR}=2.33,95 \% \mathrm{CI}=1.30-4.19, p=0.005)$, antiplatelet use $(\mathrm{OR}=4.1,95 \% \mathrm{CI}=2.16-7.77, p<0.001)$ were the factors predicting poor anticoagulant control on warfarin (TTR $<65 \%$ ) (Table 2).

In multivariate analysis, the predictive factors for poor anticoagulant control on warfarin $(\mathrm{TTR}<65 \%)$ were age $\geq 75$ years $(\mathrm{OR}=1.53,95 \% \mathrm{CI}=1.12-2.07, p=0.007)$,

Table 2 Univariate and multivariate logistic regression analysis for predictors of poor anticoagulant control on warfarin (TTR $<65 \%)$ in NVAF patients

\begin{tabular}{|c|c|c|c|c|}
\hline & \multicolumn{2}{|l|}{ Univariable analysis } & \multicolumn{2}{|c|}{ Multivariable analysis } \\
\hline & Crude odd ratio $(95 \% \mathrm{Cl})$ & $p$ value & $\begin{array}{l}\text { Adjusted odd ratio } \\
(95 \% \mathrm{Cl})\end{array}$ & $p$ value \\
\hline Age $\geq 75$ years & $1.53(1.12-2.07)$ & $0.007 \neq$ & $1.4(1.02-1.93)$ & 0.037 \\
\hline Female & $0.96(0.71-1.31)$ & 0.809 & & \\
\hline $\mathrm{BMI} \geq 25 \mathrm{~kg} / \mathrm{m}^{2}$ & $0.68(0.50-0.92)$ & $0.012 \neq$ & & \\
\hline \multicolumn{5}{|l|}{ Education } \\
\hline Primary or no & $1.48(1.02-2.16)$ & $0.041 \neq$ & & \\
\hline Secondary & $1.24(0.82-1.88)$ & 0.312 & & \\
\hline \multicolumn{5}{|l|}{ Smoking } \\
\hline Current smoker & $0.97(0.49-1.93)$ & 0.935 & & \\
\hline Ex-smoker & $0.87(0.62-1.23)$ & 0.434 & & \\
\hline Diabetes mellitus & $1.44(1.05-1.98)$ & $0.025 \neq$ & & \\
\hline Heart failure & $2.14(1.29-3.53)$ & $0.003 \neq$ & $1.7(1.01-2.86)$ & 0.047 \\
\hline Hepatic impairment & $1.64(1.06-2.52)$ & $0.025 \neq$ & & \\
\hline CKD $\left(\right.$ GFR $\left.<50 \mathrm{ml} / \mathrm{min} / 1.73 \mathrm{~m}^{2}\right)$ & $2.10(1.42-3.11)$ & $<0.001 \neq$ & $1.68(1.12-2.52)$ & 0.013 \\
\hline LVEF $<40 \%$ & $2.33(1.30-4.19)$ & $0.005 \ddagger$ & $1.92(1.04-3.53)$ & 0.037 \\
\hline Antiplatelet use & $4.10(2.16-7.77)$ & $<0.001 \neq$ & $3.49(1.82-6.66)$ & $<0.001$ \\
\hline
\end{tabular}

${ }^{\ddagger} p$ value $<0.05$ 
congestive heart failure $(\mathrm{OR}=2.14,95 \% \mathrm{CI}=1.29-3.53$, $p=0.003)$, chronic kidney disease with GFR $<50 \mathrm{ml} /$ $\mathrm{min} / 1.73 \mathrm{~m}^{2}(\mathrm{OR}=2.10,95 \% \mathrm{CI}=1.42-3.11, p<0.001)$, $\mathrm{LVEF}<40 \%(\mathrm{OR}=2.33,95 \% \mathrm{CI}=1.30-4.19, p=0.005)$, antiplatelet use $(\mathrm{OR}=4.1,95 \% \mathrm{CI}=2.16-7.77, p<0.001)$ (Table 2).

\section{The ACAChE score and the SAMe-TT2R2 score}

Based on the regression coefficients (Additional file 1: supplementary table 5), we developed the new scoring system called $\mathrm{ACAChE}$ score $[\mathrm{A}$, antiplatelet use (4 points); C, chronic kidney disease, GFR $<50 \mathrm{ml} /$ $\min / 1.73 \mathrm{~m}^{2}$ ( 2 points); A, age $\geq 75$ years (1 point);

Table 3 ACAChE score

\begin{tabular}{llc}
\hline & Definition & Score \\
\hline A & Antiplatelet use & 4 \\
C & $\begin{array}{l}\text { Chronic kidney disease, GFR }<50 \mathrm{ml} / \\
\text { min/1.73 } \mathrm{m}^{2}\end{array}$ & 2 \\
& Age $\geq 75$ years & 1 \\
Ch & History of congestive heart failure & 2 \\
E & LVEF $<40 \%$ & 2 \\
Maximum points & & 11 \\
\hline
\end{tabular}

ACAChE score $=1$ (Age $\geq 75$ years) +2 (Chf) +2 (Ckd with GFR $<50 \mathrm{ml} /$ $\left.\mathrm{min} / 1.73 \mathrm{~m}^{2}\right)+2(\operatorname{LVEF}<40 \%)+4$ (Antiplatelet use)
Ch, history of congestive heart failure (2 points); E, LVEF $<40 \%$ (2 points)] to predict poor anticoagulant control on warfarin $(\mathrm{TTR}<65 \%)$ in NVAF patients (Table 3).

The receiver-operating characteristic (ROC) curves (Fig. 2) demonstrated discrimination performance of the new scoring system (ACAChE score) in comparison with the SAMe-TT2R2 score for TTR $<65 \%$. The C-statistic of the new scoring system (ACAChE score) and the SAMe-TT2R2 score were $0.62(95 \%$ CI $0.57-0.65)$ and $0.54(95 \% \mathrm{CI} 0.50-0.58)$, respectively, with statistically significant $(p=0.007)$.

The sensitivity, specificity, PPV, NPV, positive likelihood ratio, negative likelihood ratio, and odd ratio of the new scoring system (ACAChE score) for different cutoff point to predict poor anticoagulant control on warfarin (TTR $<65 \%$ ) are shown in Table 4.

Based on the ROC curves analysis and by using Youden's index, the new scoring system (ACAChE score) at cut point $\geq 4$ was a good predictor for TTR $<65 \%$ in Thai NVAF patients [sensitivity $43.8 \%$ (95\% CI 39.9-47.8), specificity 75.2\% (95\%CI 69.1-80.7), PPV 83.3\% (95\%CI 78.8-87.1), and NPV 32.2\% (95\%CI 28.2-36.4)].

As the ACAChE score increased, the TTR decreased with statistically significant as shown in Table 5 . We categorized new scoring system (ACAChE score) with

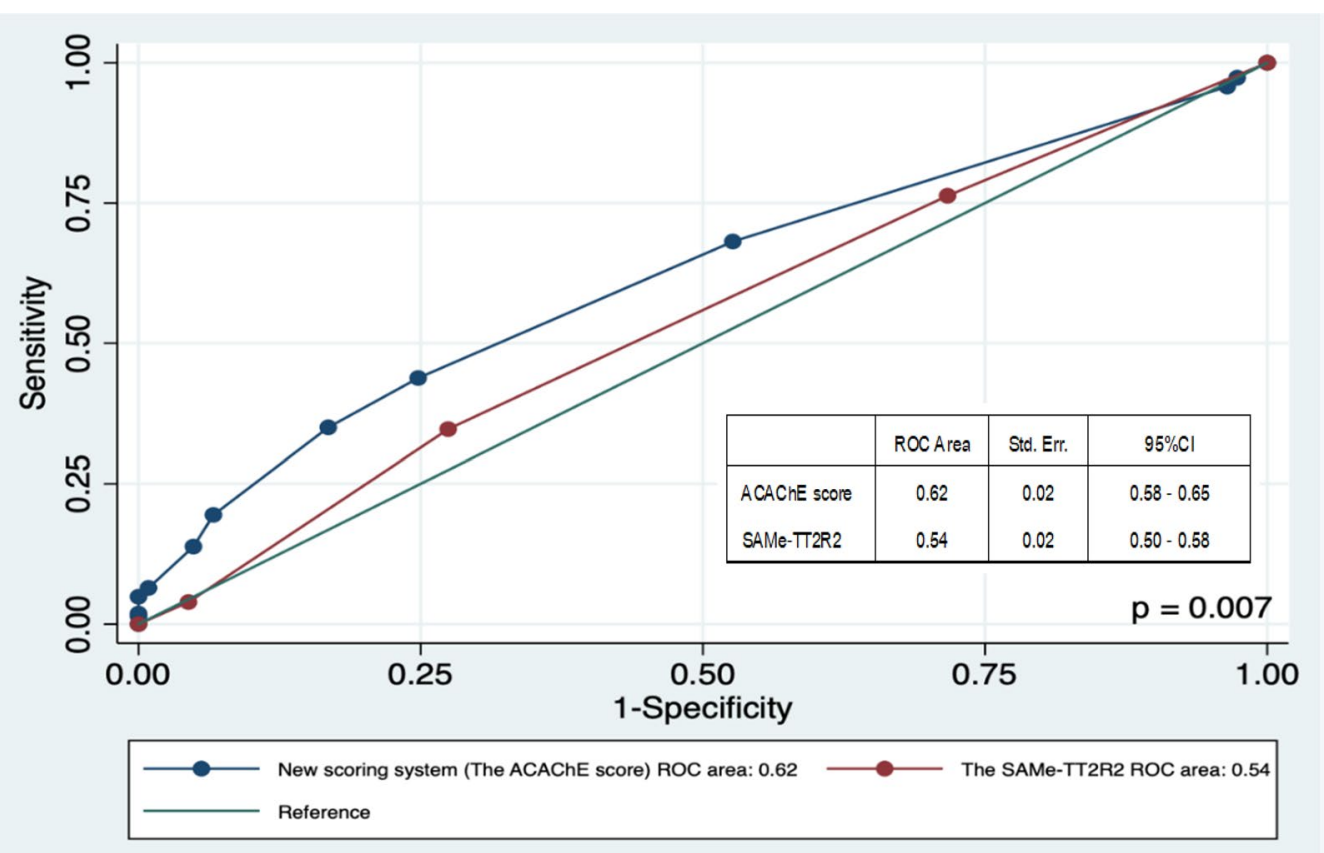

Fig. 2 Receiver-operating characteristic (ROC) curves demonstrating discrimination performance of the new scoring system (ACAChE score) in comparison with the SAMe-TT2R2 score for time in therapeutic range (TTR) $<65 \%$ 
Table 4 Different cutoff points of the ACAChE score to predict poor anticoagulant control on warfarin $(T T R<65 \%)$

\begin{tabular}{|c|c|c|c|c|c|c|c|}
\hline & $\begin{array}{l}\text { ACAChE } \\
\text { score } \geq 1 \\
\text { TTR }<65 \%\end{array}$ & $\begin{array}{l}\text { ACAChE } \\
\text { score } \geq 2 \\
\text { TTR }<65 \%\end{array}$ & $\begin{array}{l}\text { ACAChE } \\
\text { score } \geq 3 \\
\text { TTR }<65 \%\end{array}$ & $\begin{array}{l}\text { ACAChE } \\
\text { score } \geq 4 \\
\text { TTR }<65 \%\end{array}$ & $\begin{array}{l}\text { ACAChE } \\
\text { score } \geq 5 \\
\text { TTR }<65 \%\end{array}$ & $\begin{array}{l}\text { ACAChE } \\
\text { score } \geq 6 \\
\text { TTR }<65 \%\end{array}$ & $\begin{array}{l}\text { ACAChE } \\
\text { score } \geq 7 \\
\text { TTR }<65 \%\end{array}$ \\
\hline Sensitivity & $\begin{array}{l}97.3 \\
(95.8-98.4)\end{array}$ & $\begin{array}{l}95.8 \\
(93.9-97.2)\end{array}$ & $\begin{array}{l}68.1 \\
(64.4-71.7)\end{array}$ & $\begin{array}{l}43.8 \\
(39.9-47.8)\end{array}$ & $\begin{array}{l}35 \\
(31.3-38.9)\end{array}$ & $\begin{array}{l}19.5 \\
(16.5-22.8)\end{array}$ & $\begin{array}{l}13.8 \\
(11.2-16.7)\end{array}$ \\
\hline Specificity & $\begin{array}{l}2.65 \\
(0.98-5.69)\end{array}$ & $\begin{array}{l}3.54 \\
(1.54-6.86)\end{array}$ & $\begin{array}{l}47.3 \\
(40.7-54.1)\end{array}$ & $\begin{array}{l}75.2 \\
(69.1-80.7)\end{array}$ & $\begin{array}{l}83.2 \\
(77.7-87.8)\end{array}$ & $\begin{array}{l}93.4 \\
(89.3-96.2)\end{array}$ & $\begin{array}{l}95.1 \\
(91.5-97.5)\end{array}$ \\
\hline $\begin{array}{l}\text { Likelihood ratio } \\
\qquad(+)\end{array}$ & $\begin{array}{l}1.00 \\
(0.975-1.03)\end{array}$ & $\begin{array}{l}0.993 \\
(0.964-1.02)\end{array}$ & $\begin{array}{l}1.29 \\
(1.13-1.48)\end{array}$ & $\begin{array}{l}1.77 \\
(1.39-2.26)\end{array}$ & $\begin{array}{l}2.08 \\
(1.53-2.83)\end{array}$ & $\begin{array}{l}2.93 \\
(1.75-4.9)\end{array}$ & $\begin{array}{l}2.84 \\
(1.55-5.21)\end{array}$ \\
\hline $\begin{array}{l}\text { Likelihood ratio } \\
\qquad(-)\end{array}$ & $\begin{array}{l}1.01 \\
(0.401-2.52)\end{array}$ & $\begin{array}{l}1.2 \\
(0.552-2.6)\end{array}$ & $\begin{array}{l}0.673 \\
(0.563-0.804)\end{array}$ & $\begin{array}{l}0.747 \\
(0.675-0.827)\end{array}$ & $\begin{array}{l}0.781 \\
(0.72-0.848)\end{array}$ & $\begin{array}{l}0.863 \\
(0.819-0.908)\end{array}$ & $\begin{array}{l}0.906 \\
(0.868-0.946)\end{array}$ \\
\hline Odd ratio & $\begin{array}{l}0.995 \\
(0.399-2.48)\end{array}$ & $\begin{array}{l}0.829 \\
(0.378-1.82)\end{array}$ & $\begin{array}{l}1.92 \\
(1.41-2.62)\end{array}$ & $\begin{array}{l}2.37 \\
(1.69-3.32)\end{array}$ & $\begin{array}{l}2.66 \\
(1.82-3.91)\end{array}$ & $\begin{array}{l}3.4 \\
(1.96-5.91)\end{array}$ & $\begin{array}{l}3.13 \\
(1.66-5.92)\end{array}$ \\
\hline $\begin{array}{l}\text { Positive predic- } \\
\text { tive value }\end{array}$ & $\begin{array}{l}73.8 \\
(70.7-76.8)\end{array}$ & $\begin{array}{l}73.7 \\
(70.5-76.6)\end{array}$ & $\begin{array}{l}78.5 \\
(74.8-81.8)\end{array}$ & $\begin{array}{l}83.3 \\
(78.8-87.1)\end{array}$ & $\begin{array}{l}85.4 \\
(80.6-89.5)\end{array}$ & $\begin{array}{l}89.2 \\
(82.8-93.8)\end{array}$ & $\begin{array}{l}88.9 \\
(81-94.3)\end{array}$ \\
\hline $\begin{array}{l}\text { Negative predic- } \\
\text { tive value }\end{array}$ & $\begin{array}{l}26.1 \\
(10.2-48.4)\end{array}$ & $\begin{array}{l}22.9 \\
(10.4-40.1)\end{array}$ & $\begin{array}{l}34.5 \\
(29.2-40.1)\end{array}$ & $\begin{array}{l}32.2 \\
(28.2-36.4)\end{array}$ & $\begin{array}{l}31.2 \\
(27.5-35.1)\end{array}$ & $\begin{array}{l}29.1 \\
(25.9-32.6)\end{array}$ & $\begin{array}{l}28.1 \\
(25-31.5)\end{array}$ \\
\hline Accuracy & $\begin{array}{l}72.54 \\
(69.43-75.49)\end{array}$ & $\begin{array}{l}71.61 \\
(68.47-74.6)\end{array}$ & $\begin{array}{l}62.69 \\
(59.36-65.93)\end{array}$ & $\begin{array}{l}52.03 \\
(48.63-55.41)\end{array}$ & $\begin{array}{l}47.62 \\
(47.71-54.49)\end{array}$ & $\begin{array}{l}38.82 \\
(35.55-42.16)\end{array}$ & $\begin{array}{l}35.11 \\
(31.92-38.4)\end{array}$ \\
\hline$p$ value & 0.991 & 0.647 & $<0.001$ & $<0.001$ & $<0.001$ & $<0.001$ & $<0.001$ \\
\hline
\end{tabular}

cutoff point at 3 . The NVAF patients with ACAChE score $\geq 4$ had significantly reduced in mean TTR compared with ACAChE score 0-3 as shown in Table 5.

Comparison between different level of SAMe-TT2R2 score had no statistically significance (Table 5). This study categorized patients into two groups including high risk and low risk group by using different cutoff point. SAMeTT2R2 score A used cutoff point at 2 and SAMeTT2R2 score B used cutoff point at 3 . The SAMeTT2R2 score B showed statistically significant difference in mean TTR, but the SAMeTT2R2 score A had no statistically significant difference in mean TTR as shown in Fig. 3.

The sensitivity, specificity, PPV, NPV, positive likelihood ratio, negative likelihood ratio, and odd ratio of the SAMe-TT2R2 score for different cutoff point to predict poor anticoagulant control on warfarin $(\mathrm{TTR}<65 \%)$ are shown in Additional file 1: supplementary Table 6 . The NVAF patients with TTR $<65 \%$ who had SAMe-TT2R2 score $2,3,4$, and $\geq 5$ were $25 \%$, $42.3 \%, 28.7 \%$, and $4 \%$, respectively.

Based on the ROC curves analysis, the best cutoff point of the SAMe-TT2R2 score for Thai population to predict poor anticoagulation control on warfarin (TTR $<65 \%$ ) as based on our study was $\geq 4$ [sensitivity $34.7 \%$ (95\%CI $31.0-38.5$ ), specificity $72.7 \%$ (95\%CI 66.4-78.4), PPV 78.1\% (95\%CI 72.8-82.8), NPV $28.4 \%$ (95\%CI 24.8-32.3)]).

\section{Discussion}

The main principal results of this study showed that age $\geq 75$ years, congestive heart failure, chronic kidney disease with GFR $<50 \mathrm{ml} / \mathrm{min} / 1.73 \mathrm{~m}^{2}$, left ventricular ejection fraction $<40 \%$, antiplatelet use were predictive factors for poor anticoagulant control on warfarin $(\mathrm{TTR}<65 \%)$ in Thai NVAF patients. The new scoring system (ACAChE score) had better discrimination performance for predicting poor anticoagulant control on warfarin $(\mathrm{TTR}<65 \%)$ in Thai NVAF patients than the SAMe-TT2R2 score. The best cutoff point for the new scoring system (ACAChE score) by using Youden's index was $\geq 4$ [sensitivity $43.8 \%$ (95\%CI 39.9-47.8), specificity 75.2\% (95\%CI 69.1-80.7), PPV 83.3\% (95\%CI 78.8-87.1), and NPV $32.2 \%(95 \%$ CI 28.2-36.4)]. The SAMe-TT2R2 score was not good enough for discriminate poor anticoagulant control on warfarin $(\mathrm{TTR}<65 \%)$ in Thai NVAF patients due to the low C-statistic [0.54 (95\%CI 0.50 0.58)]. Our study also searched for the best cutoff point for the SAMe-TT2R2 in Thai NVAF population and the best cutoff point based on ROC curve analysis was $\geq 4$ [sensitivity $34.7 \%$ (95\%CI 31.0-38.5), specificity $72.7 \%$ (95\%CI 66.4-78.4), PPV 78.1\% (95\%CI 72.8-82.8), NPV $28.4 \%$ (95\%CI 24.8-32.3)].

In 2013, Apostolakis et al. [10] concluded that female, non-Caucasian, age $<60$ years, smoking, having more than two comorbidities and being treated with amiodarone were the factors associated with suboptimal 
anticoagulant control. But in our study, the result was not correlated with Apostolakis et al. [10]. It showed that older patients had lower TTR than younger patients. This might be due to multiple comorbidities in older patients such as CKD and liver disease, taking polypharmacy, poor compliance due to taking polypharmacy, herbal use and poor understanding in diet restriction which interacted with warfarin and contributed to lower TTR. Chronic kidney disease, heart failure and left ventricular ejection fraction $<40 \%$ were the factors that associated with poor anticoagulant control on warfarin in our study, which was similarly to the previous study [10]. To our knowledge, our study was the first that proposed the association between antiplatelet use and poor anticoagulant control on warfarin (TTR $<65 \%)(\mathrm{OR}=4.1,95 \%$ $\mathrm{CI}=2.16-7.77, p<0.001)$. Antiplatelet interfered with warfarin metabolism with a subsequent increase in INR, which contributed to lower TTR. Concomitant therapy with warfarin and antiplatelet had shown significantly increase risk of minor and major bleeding [17].

The safety and efficacy of warfarin for stroke prevention in NVAF depended on TTR $[9,10]$. The TTR $<65 \%$ was associated with poor anticoagulant control on warfarin and several studies reported that Asian population had lower TTR than Western population $[9,18]$. From the GARFIELD-AF registry, $16.7 \%$ of NVAF patients in Asian countries had TTR $\geq 65 \%$ and $49.4 \%$ of NVAF patients in European countries had TTR $\geq 65 \%$ [9]. In our study, 26\% of NVAF patients had TTR $>65 \%$ which is better than the
Asian data reporting in GARFIELD-AF registry and the mean TTR of this study was $48.11 \pm 25.18 \%$ which was lower than the Western population from GARFIELD-AF registry. The possible reason that our study had higher TTR than in Asian data from GARFIELD-AF registry might be due to more frequent INR measurement in patients with SAMe-TT2R2 > 2, as in recommendation in recent guidelines for NVAF patients who received warfarin $[1,5-7]$.

In Chinese population, Pak Hei Chan et al. [18] reported average TTR in Asian population was $38.2 \pm 24.4 \%$. The average TTR in our study was $48.11 \pm 25.18 \%$ which is lower than the Western population from GARFIELD-AF registry [9] and lower than recent guidelines recommendation $[1,5-7]$. This result was similar to recent studies $[9,17]$. The low TTR in Asian populations may be from the genetic factors on polymorphisms of cytochrome P450 2C9 (CYP2C9) and vitamin $\mathrm{K}$ epoxide reductase complex subunit 1 (VKORC1) which affected to variation in amount of warfarin dose [19]. 95.2\% of Thai population had CYP2C9 "1/"1 genotype, and $63.2 \%$ of Thai population also had VKORC1 genotype. However, it remained unclear in the influence degree of genetic factors effect on poor anticoagulant control on warfarin in Asian population. The risk of intracerebral hemorrhage in Asian population who treated with warfarin was four times higher than in Western population; thus, some physicians preferred to lower the target of INR than recommendation, representing the main reason for lower

Table 5 TTR in relation to the SAMe TT2R2 and new scoring system (ACAChE score)

\begin{tabular}{|c|c|c|c|c|c|c|}
\hline & \multicolumn{3}{|c|}{ The SAMe TT2R2 } & \multicolumn{3}{|c|}{ New scoring system (ACAChE score) } \\
\hline & $n(\%)$ & $\begin{array}{l}\text { TTR (\%): } \\
\text { Mean } \pm \text { SD }\end{array}$ & $p$ value & $n(\%)$ & $\begin{array}{l}\text { TTR (\%): } \\
\text { Mean } \pm \text { SD }\end{array}$ & $p$ value \\
\hline 0 & & & 0.224 & $23(2.66)$ & $45.99 \pm 27.39$ & $<0.001$ \\
\hline 1 & & & & $12(1.39)$ & $48.37 \pm 23.89$ & \\
\hline 2 & $216(24.89)$ & $48.13 \pm 28.07$ & & $276(31.94)$ & $51.53 \pm 27.38$ & \\
\hline 3 & $365(42.12)$ & $46.35 \pm 26.10$ & & $218(25.23)$ & $48.10 \pm 25.45$ & \\
\hline 4 & $248(28.65)$ & $42.93 \pm 26.13$ & & $74(8.57)$ & $41.71 \pm 26.03$ & \\
\hline 5 & $27(3.42)$ & $46.13 \pm 27.30$ & & $122(14.12)$ & $43.69 \pm 25.16$ & \\
\hline 6 & $8(0.91)$ & $36.25 \pm 26.45$ & & $40(4.63)$ & $32.88 \pm 25.00$ & \\
\hline 7 & & & & $56(6.48)$ & $39.50 \pm 24.86$ & \\
\hline 8 & & & & $12(1.39)$ & $44.63 \pm 31.55$ & \\
\hline 9 & & & & $19(2.20)$ & $23.33 \pm 19.95$ & \\
\hline 10 & & & & $3(0.34)$ & $10.13 \pm 8.81$ & \\
\hline 11 & & & & $9(1.04)$ & $20.37 \pm 17.11$ & \\
\hline \multicolumn{7}{|c|}{ Cut off } \\
\hline $0-2$ & 216 (24.89) & $48.13 \pm 28.07$ & 0.124 & $311(35.00)$ & $50.99 \pm 27.22$ & $<0.001$ \\
\hline$\geq 3$ & $648(75.11)$ & $44.91 \pm 26.18$ & & $553(64.00)$ & $42.72 \pm 25.94$ & \\
\hline $0-3$ & $581(67.01)$ & $47.01 \pm 26.84$ & 0.040 & $529(61.23)$ & $49.80 \pm 26.52$ & $<0.001$ \\
\hline$\geq 4$ & 283 (32.99) & $43.05 \pm 26.20$ & & 335 (38.77) & $39.21 \pm 25.69$ & \\
\hline
\end{tabular}




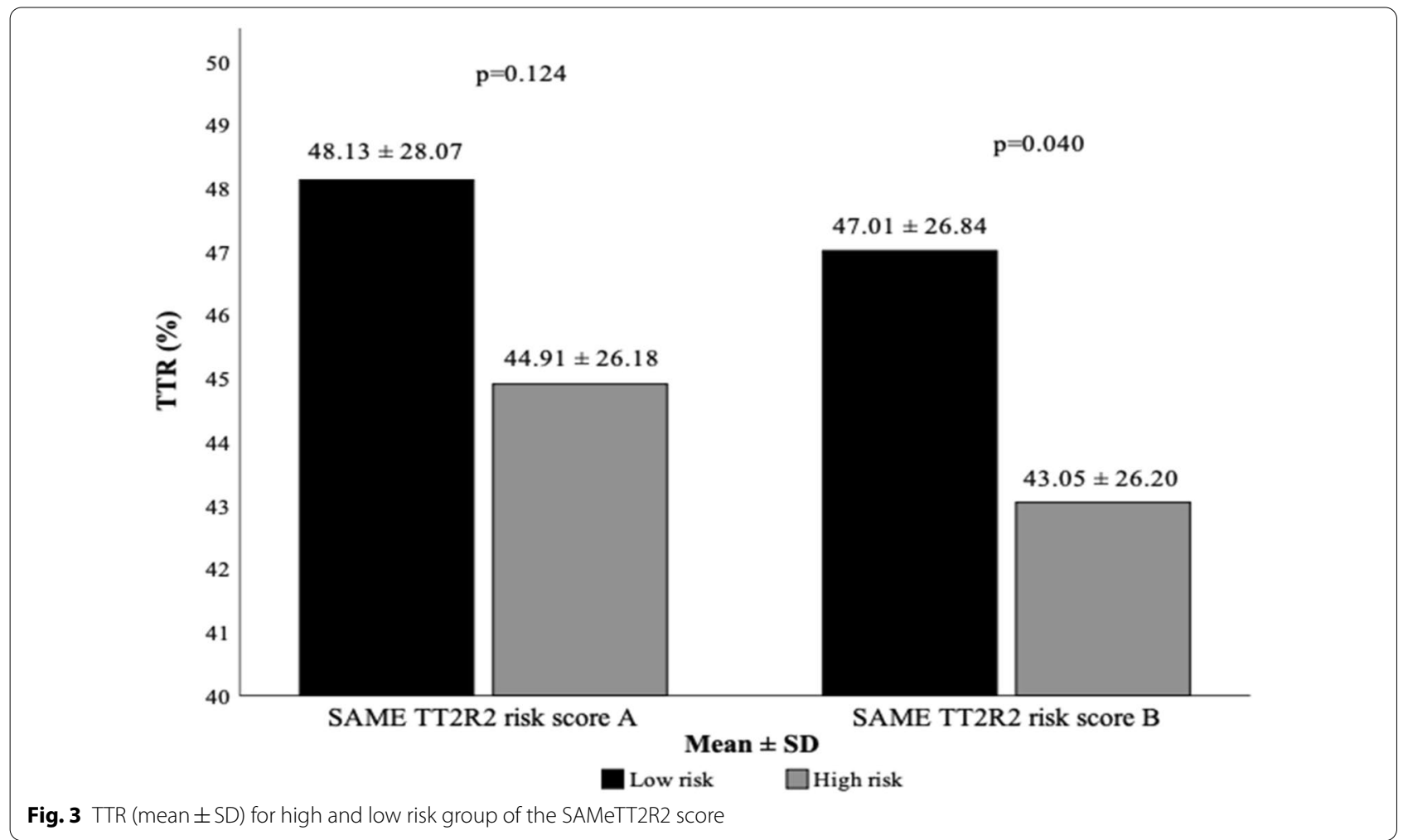

TTR in Asian population $[5,8,18]$. Also in Asian countries, herbal medication was also one of the reason that affected TTR in NVAF patients on warfarin.

In 2013, Apostolakis et al. [10] proposed the SAMeTT2R2 score for prediction of the quality of anticoagulant control (as reflected by TTR) and expected to guide the selection of oral anticoagulation. The SAMe-TT2R2 score in the range of 0 to 2 predicts the effect of good anticoagulation control (TTR $\geq 65 \%$ ) and the use of warfarin would be acceptable. In 2016, Pak Hei Chan et al. [20] reported use of the SAMe-TT2R2 score for prediction of good anticoagulation control on warfarin in Chinese patients with atrial fibrillation. Patient with SAMe-TT2R2 score $>2$ points predicted poor anticoagulant control (TTR $<70 \%)$. However, it was found that the average TTR in the non-Caucasian Chinese population was $38.2 \pm 24.4 \%$. It recommended that patients with the SAMe-TT2R2 score $>2$ should have regular INR check and preferred the selection of NOACs over warfarin because the SAMe-TT2R2 score $>2$ associated with poor anticoagulation control on warfarin $[1,5]$. However, the SAMe-TT2R2 score already regarded Asian as important risk factors for 2 points on the basis of ethnicity, this might overestimate the number of the NVAF patients in Asian countries who had poor anticoagulation control on warfarin.
The SAMe-TT2R2 score has not been much validated in Asian population. Two recent studies from Pak Hei Chan et al. [20] and Bernaitis et al. [21] had validate SAMe-TT2R2 score in Asian population. From Pak Hei Chan et al. [20], the SAMe-TT2R2 2 score $>2$ predicted poor anticoagulant control on warfarin $(\mathrm{TTR}<70 \%)$ and had association with increased risk of stroke. Our study is the third study that validates SAMe-TT2R2 score in Asian population. Previous ten studies reported that the SAMe-TT2R2 score had C-statistic of $0.52-0.72$ for prediction of poor anticoagulant control on warfarin [19]. In our study, the SAMe-TT2R2 score for predicting TTR $<65 \%$ in NVAF patients had C-statistic 0.54 (95\% CI 0.50-0.58); thus, the SAMe-TT2R2 was a score that had low capability to predict poor anticoagulation control on warfarin in Thai NAVF patients. Therefore, use of the SAMe-TT2R2 score in clinical practice is limited because the discrimination power is low and cannot be applied to Asian population. Thus, our study developed the new scoring system (ACAChE score) which showed moderate capability to predict poor anticoagulation control on warfarin $(\mathrm{TTR}<65 \%)$ and had better discrimination performance for predicting poor anticoagulant control on warfarin $(\mathrm{TTR}<65 \%)$ in Thai NVAF patients than the SAMe-TT2R2 score. This study showed the best cutoff point for the new scoring system (ACAChE score) $\geq 4$ was a good predictor for TTR $<65 \%$ in Thai NVAF 
patients [sensitivity 43.8\% (95\%CI 39.9-47.8), specificity 75.2\% (95\%CI 69.1-80.7), PPV 83.3\% (95\%CI 78.8-87.1), and NPV 32.2\% (95\%CI 28.2-36.4)]. After we categorized new scoring system (ACAChE score) with cutoff point at 3 , the NVAF patients with ACAChE score $\geq 4$ had significantly lower mean TTR than in group of ACAChE score $0-3$. This showed that ACAChE score had ability to predict poor anticoagulant control on warfarin in NVAF patients $(\mathrm{TTR}<65 \%)$. Patients with ACAChE score $\geq 4$ might need more frequent INR measurement, frequent follow-up, educational program. Thus, our study expected that the new scoring system (ACAChE score) could guide the selection of oral anticoagulation.

Interestingly, the SAMe-TT2R2 score may overestimate patients who had TTR $<65 \%$ due to regarding being Asians as a risk factor (2 points), so the cutoff point for the SAMe-TT2R2 score in Asian population may be higher than Western population to predict poor anticoagulant control on warfarin in NVAF patients. This study also revealed the new best cutoff point for the SAMe-TT2R2 score in Asian population which was different from the previous study [10]. The new best cutoff point for the SAMe-TT2R2 was $>3$ [sensitivity $34.7 \%$ (95\%CI 31.0-38.5), specificity 72.7\% (95\%CI 66.4-78.4), PPV 78.1\% (95\%CI 72.8-82.8), NPV 28.4\% (95\%CI 24.832.3)], which was higher than Pak Hei Chan et al. [17] and Bernaitis et al. [18] studies.

For the clinical implication, the new scoring system (ACAChE score) could aid decision making for oral anticoagulation. Thai NVAF patients with ACAChE score $0-3$ would do well on warfarin, or conversely, with ACAChE score $\geq 4$ would have poor anticoagulant control on warfarin and might require more frequent INR measurement, frequent follow-up, educational program or using NOACs to achieve good oral anticoagulant control. We expected that ACAChE score would help low- to middle-income Asian countries (which cost-effectiveness is an issue) in selection of proper oral anticoagulation.

\section{Limitation}

This study was a single-center retrospective study and study population in our study were all from King Chulalongkorn Memorial Hospital which is a tertiary care hospital. Therefore, it might not be extrapolated to the NVAF patients in primary and secondary care hospitals. Another limitation was that the new scoring system (ACAChE score) had not been validated in external cohort. Our study had not shown the clinical outcomes data, and thus we could not correlate the relationship of the new scoring system (ACAChE score) and TTR with clinical outcomes.

\section{Conclusion}

In Thai NVAF patients, the ACAChE score (antiplatelet use, chronic kidney disease (GFR $<50 \mathrm{ml} / \mathrm{min} / 1.73 \mathrm{~m}^{2}$ ), age $\geq 75$ years, history of congestive heart failure, and LVEF $<40 \%$ ) had better prediction for TTR $<65 \%$ than SAMe-TT2R2 score. Thus, it expected to guide the selection of oral anticoagulation in low- to middle-income countries in Asia.

\section{Supplementary Information}

The online version contains supplementary material available at https://doi. org/10.1186/s42444-021-00038-8.

Additional file 1.

\section{Acknowledgements}

Thanks to the financial support from division of Cardiology, faculty of medicine, Chulalongkorn University and King Chulalongkorn Memorial Hospital

\section{Authors' contributions}

All authors reviewed and approved the final manuscript.

Funding

The authors received no specific funding for this work.

Availability of supporting data

The authors confirm that the data supporting the findings of this study are available within the article and its supplementary materials.

\section{Declarations}

Ethical approval and consent to participate

The study was approved by the institutional review board, Faculty of Medicine, Chulalongkorn University, Bangkok, Thailand (IRB Number 105/62).

\section{Consent for publication}

Not applicable.

\section{Competing interests}

All authors declare no conflict of interest, no plagiarism, no fabrication, and no falsification.

Received: 18 February 2021 Accepted: 14 May 2021

Published online: 14 June 2021

References

1. Kirchhof P, Benussi S, Kotecha D, Ahlsson A, Atar D, Casadei B, et al. 2016 ESC guidelines for the management of atrial fibrillation developed in collaboration with EACTS. Eur Heart J. 2016;37(38):2893-962.

2. Miller CS, Grandi SM, Shimony A, Filion KB, Eisenberg MJ. Meta-analysis of efficacy and safety of new oral anticoagulants (dabigatran, rivaroxaban, apixaban) versus warfarin in patients with atrial fibrillation. Am J Cardiol. 2012;110(3):453-60.

3. Ruff CT, Giugliano RP, Braunwald E, Hoffman EB, Deenadayalu N, Ezekowitz $M D$, et al. Comparison of the efficacy and safety of new oral anticoagulants with warfarin in patients with atrial fibrillation: a meta-analysis of randomised trials. Lancet. 2014;383(9921):955-62.

4. Deitelzweig S, Farmer C, Luo X, Li X, Vo L, Mardekian J, et al. Comparison of major bleeding risk in patients with non-valvular atrial fibrillation receiving direct oral anticoagulants in the real-world setting: a network meta-analysis. Curr Med Res Opin. 2018;34(3):487-98. 
5. Chiang CE, Okumura K, Zhang S, Chao TF, Siu CW, Wei Lim T, et al. 2017 consensus of the Asia Pacific Heart Rhythm Society on stroke prevention in atrial fibrillation. J Arrhythm. 2017;33(4):345-67.

6. January CT, Wann LS, Alpert JS, Calkins H, Cigarroa JE, Cleveland JC Jr, et al. 2014 AHA/ACC/HRS guideline for the management of patients with atrial fibrillation: a report of the American College of Cardiology/American Heart Association Task Force on Practice Guidelines and the Heart Rhythm Society. J Am Coll Cardiol. 2014;64(21):e1-76.

7. January CT, Wann LS, Calkins H, Chen LY, Cigarroa JE, Cleveland JC Jr, et al. 2019 AHA/ACC/HRS focused update of the 2014 AHA/ACC/HRS guideline for the management of patients with atrial fibrillation. Circulation. 2019;140(2):e125-51.

8. Tse HF, Wang YJ, Ahmed Ai-Abdullah M, Pizarro-Borromeo AB, Chiang CE, Krittayaphong $\mathrm{R}$, et al. Stroke prevention in atrial fibrillation-an Asian stroke perspective. Heart Rhythm. 2013;10(7):1082-8.

9. Haas S, Ten Cate H, Accetta G, Angchaisuksiri P, Bassand JP, Camm AJ, et al. Quality of vitamin Kantagonist control and 1-year outcomes in patients with atrial fibrillation: a global perspective from the GARFIELD-AF registry. PLoS ONE. 2016;11(10):e0164076.

10. Gallagher AM, Setakis E, Plumb JM, Clemens A, van Staa TP. Risks of stroke and mortality associated with suboptimal anticoagulation in atrial fibrillation patients. Thromb Haemost. 2011;106(5):968-77.

11. Apostolakis S, Sullivan RM, Olshansky B, Lip GYH. Factors affecting quality of anticoagulation control among patients with atrial fibrillation on warfarin: the SAMe-TT(2)R(2) score. Chest. 2013;144(5):1555-63.

12. Pivatto Junior F, Scheffel RS, Ries L, Wolkind RR, Marobin R, Barkan SS, et al. SAMe-TT2R2 score in the outpatient anticoagulation clinic to predict time in therapeutic range and adverse events. Arq Bras Cardiol. 2017;108(4):290-6

13. Poli D, Antonucci E, Testa S, Lip GY. A prospective validation of the SAMETT2R 2 score: how to identify atrial fibrillation patients who will have good anticoagulation control on warfarin. Intern Emerg Med. 2014;9(4):443-7.
14. van Miert JHA, Bos S, Veeger N, Meijer K. Clinical usefulness of the SAMeTT2R2 score: a systematic review and simulation meta-analysis. PLOS ONE. 2018;13(3):e0194208.

15. Proietti M, Lane DA, Lip GY. Relation of the SAMe-TT2R2 score to quality of anticoagulation control and thromboembolic events in atrial fibrillation patients: observations from the SPORTIF trials. Int J Cardiol. 2016;216:168-72.

16. Roldan V, Cancio S, Galvez J, Valdes M, Vicente V, Marin F, et al. The SAMeTT2R2 score predicts poor anticoagulation control in AF patients: a prospective"real-world" inception cohort study. Am J Med. 2015;128(11):1237-43.

17. Delaney JA, Opatrny L, Brophy JM, Suissa S. Drug drug interactions between antithrombotic medications and the risk of gastrointestinal bleeding. CMAJ. 2007;177(4):347-51.

18. Chiang CE, Wang KL, Lip GY. Stroke prevention in atrial fibrillation: an Asian perspective. Thromb Haemost. 2014;111(5):789-97.

19. Zulkifly H, Lip GYH, Lane DA. Use of the SAMe-TT2R2 score to predict anticoagulation control in atrial fibrillation and venous thromboembolism patients receiving vitamin K antagonists: a review. Heart Rhythm. 2018;15(4):615-23.

20. Chan PH, Hai JJ, Chan EW, Li WH, Tse HF, Wong IC, et al. Use of the SAMeTT2R2 score to predict good anticoagulation control with warfarin in Chinese patients with atrial fibrillation: relationship to ischemic stroke incidence. PLoS ONE. 2016;11(3):e0150674.

21. Bernaitis N, Ching CK, Chen L, Hon JS, Teo SC, Davey AK, et al. The sex, age, medical history, treatment, tobacco use, race risk (SAMe TT2R2) score predicts warfarin control in a Singaporean population. J Stroke CerebrovasC Dis Off J Natl Stroke Assoc. 2017;26(1):64-9.

\section{Publisher's Note}

Springer Nature remains neutral with regard to jurisdictional claims in published maps and institutional affiliations.
Ready to submit your research? Choose BMC and benefit from:

- fast, convenient online submission

- thorough peer review by experienced researchers in your field

- rapid publication on acceptance

- support for research data, including large and complex data types

- gold Open Access which fosters wider collaboration and increased citations

- maximum visibility for your research: over $100 \mathrm{M}$ website views per year

At BMC, research is always in progress.

Learn more biomedcentral.com/submissions 